\title{
«Treschevaleureux capitaines » contre « crocheteurs de flascons » : lecteurs et lectorats dans les Fascetieux devitz des Cent nouvelles nouvelles du Seigneur de La Motte Roullant
}

\author{
NORA VIET
}

Université Clermont-Auvergne

Le récit bref de la Renaissance doit en partie son succès à son ubiquité sociale : fables, fabliaux, nouvelles circulaient dans les sphères les plus diverses, de la cour aux cercles humanistes, des milieux bourgeois au clergé. Avec la vogue du recueil de nouvelles dans les années 1540-1550, on voit pourtant s'afficher, dans les péritextes, des figures de lecteurs littérairement et socialement marquées, parfois explicitement opposées entre elles: lecteurs bourgeois et lecteurs de cour, honnêtes dames et gentilshommes, amateurs de nouvelles à l'italienne et lecteurs de contes gaulois. L'article se propose d'analyser ces clivages à partir d'un recueil où ils s'expriment avec un relief particulier: les Fascetieux devitz des Cent nouvelles nouvelles, imprimés à Lyon sous le nom d’auteur de La Motte Roullant (1549). La représentation d'un lectorat différencié et haut en couleur participe ici de lélection d'un public de nouvelles à la française.

During the Renaissance, the short narrative owes its success partly to its social ubiquity: fables, fabliaux, and short stories circulated in very different realms, from the court to humanist circles, from bourgeois society to the clergy. However, with the popularity of the short story collection between 1540 and 1550, we see their peripheral texts (peritexts) begin to feature readerly figures with a marked literary and social significance, sometimes explicitly opposed to one another: bourgeois readers and courtly readers, respectable women and gentlemen, amateurs of Italian-style novellas and readers of bawdy tales. This article offers an analysis of these divisions on the basis of a collection where they are particularly salient: the Fascetieux devitz des Cent nouvelles nouvelles printed in Lyon under the name La Motte Roullant (1549). Here, the representation of a differentiated and highly colourful readership plays a part in the definition of an audience for French-style nouvelles.

T e récit bref de la Renaissance doit en partie son succès à son ubiquité sociale : Lfables, facéties et nouvelles circulaient à la Renaissance dans les sphères les plus diverses, de la cour aux cercles humanistes, des milieux bourgeois au clergé, empruntant tour à tour les chemins de la transmission orale et les voies de la médiation écrite ${ }^{1}$.

1. Il faut y ajouter la pratique de la lecture à haute voix, dont l'importance au XVI siècle a notamment été mise en lumière par Roger Chartier, Lectures et lecteurs dans la France d'ancien régime (Paris: Seuil, 1987). 
Cette extrême " mobilité $»^{2}$ des récits n'exclut pas pour autant qu'une différenciation générique, stylistique et éditoriale ne se manifeste petit à petit dans les recueils qui réunissaient les anecdotes, et que celle-ci ne participe, de manière plus ou moins explicite, à l'élection de publics distincts. De fait, la vogue éditoriale du recueil de nouvelles dans les années 1540-1550, « âge d'or » de la nouvelle selon l'historiographie littéraire ${ }^{3}$, va de pair avec une diversification de la production et avec un étoffement du péritexte, lequel, à travers les privilèges, pages de titres, dédicaces et épîtres aux lecteurs, fait une place croissante à la désignation d'un lectorat différencié. Cette désignation du lecteur pouvait prendre des formes diverses, perpétuant volontiers, d'ailleurs, les modes de destination du livre médiéval. Alors que les épîtres dédicatoires adressées à tel mécène ou commanditaire désignaient un lecteur unique historiquement incarné, prologues et épîtres au lecteur dessinaient les contours de ce lecteur « idéal $»^{4}$, « virtuel $»^{5}$ ou « implicite $»^{6}$ théorisé au cours des dernières décennies dans le cadre de l'analyse du discours, auquel l'acheteur du livre était invité à s'identifier $^{7}$. La fonction de ce lecteur idéal, on le sait, était en grande partie promotionnelle et servait le plus souvent de support à un discours sur l'œuvre qui cherchait à en expliciter les enjeux et à en orienter la réception. Il reste que ce lecteur "de papier » pouvait revêtir des traits socio-historiques plus ou moins précis, et inviter ainsi le lecteur « réel » à associer telle tradition

2. Jelle Koopmans voit dans cette «mobilité » de l'anecdote l'une de ses caractéristiques les plus fondamentales. Voir « Du bon mot à la mauvaise plaisanterie : le monde des anecdotes », in Traduire le mot d'esprit : pour une géographie du rire à la Renaissance, éd. Nora Viet (Paris : Classiques Garnier, à paraître).

3. Par exemple Gabriel-André Pérouse, Nouvelles françaises au $X V^{e}$ siècle. Images de la vie du temps (Genève : Droz, 1977), chapitre 5 : «Le foisonnement d'un âge d'or » (1535-1545), 100-118.

4. Wolfgand Iser, L'Acte de lecture. Théorie de l'effet esthétique, trad. Evelyne Sznycer (Bruxelles : Pierre Mardaga, 1985), 62-63. Pour une mise en perspective de ces concepts appliqués à la question des lecteurs de Rabelais, voir Nicolas Le Cadet, “"Beuveurs tresillustres, et vous verolez tresprecieux” : Rabelais et les anagnostes ", Revue d'Histoire littéraire de la France 115 (2015) : 261-282.

5. Didier Coste, "Trois conceptions du lecteur et leur contribution à une théorie du texte littéraire ", Poétique 43 (septembre 1980) : 354-371.

6. Iser, L'Acte de lecture, 62-63.

7. Les épîtres dédicatoires désignant un destinataire historiquement incarné pouvaient également susciter l'identification d'un lectorat plus large, mais le processus d'identification était alors indirect. La dédicace assumait alors souvent une fonction promotionnelle et mettait en valeur l'édition. Cet emploi stratégique de la dédicace a pu être étudié, par exemple, chez le libraire parisien Antoine Vérard ; voir Mary Beth Winn, Anthoine Vérard: Parisian Publisher. Prologues, Poems and Presentations (Genève : Droz, 1997). 
littéraire et tel type de lectorat. Or, si la différenciation sociale du lectorat de nouvelles semble a priori devoir s'envisager en termes de complémentarité et de successivité - on sait que les recueils narratifs circulaient dans des milieux différents au prix de rééditions successives ${ }^{8}-$, il est frappant de constater que c'est souvent de manière clivée ou concurrentielle que sont évoqués ces différents lectorats dans les péritextes. On voit ainsi poindre, sinon une tension, $\mathrm{du}$ moins une sorte de concurrence entre lecteurs bourgeois et lecteurs de cour, honnêtes dames et gentilshommes, amateurs de nouvelles à l'italienne et lecteurs de contes gaulois.

On analysera ici ces clivages à partir d'une édition qui les reflète et les condense de manière particulièrement saisissante, savoureuse et ludique : les Fascetieux devitz des Cent nouvelles nouvelles, imprimées en 1549 pour le libraire parisien Jean Réal, sous le nom d'auteur de "seigneur de La Motte Roullant Lyonnais ». Le péritexte de cette édition est en effet révélateur des clivages que l'on observe ailleurs, à cette période, dans le champ narratif, et montre de manière exemplaire comment la mise en scène de ces tensions contribue à l'élection et à la construction d'un public de nouvelles français.

\section{Genèse éditoriale d'une compilation à succès}

Les Fascetieux devitz des Cent nouvelles nouvelles paraissent en 1549, à Paris, simultanément chez les libraires Jean Le Bret et Jean Réal, avec Privilège du roi, sous un titre qui en annonce d'emblée la portée et en trahit indirectement la source: Les Fascetieux devitz des cent nouvelles, nouvelles, tres recreatives et fort exemplaires pour resveiller les bons espritz Françoys, veuz et remis en leur naturel, par le seigneur de la Motte Roullant Lyonnois, homme tresdocte \& bien renommés.

Gabriel-André Pérouse ${ }^{10}$ déjà avait identifié ces Fascetieux devitz comme une réécriture des Cent nouvelles nouvelles dites bourguignonnes ${ }^{11}$, composées

8. Il en était ainsi des éditions préparées par le libraire parisien Antoine Vérard pour différents commanditaires illustres, dont Charles VIII lui-même. Certaines éditions étaient imprimées sur vélin et richement enluminées, d'autres étaient de facture plus simple, imprimées sur papier, à destination d'un public plus large, en particulier de la bourgeoisie parlementaire du Palais. Voir Winn, Anthoine Vérard.

9. Paris : Jean Le Bret, 1549 et Paris : Jean Réal, 1549.

10. Pérouse, Nouvelles françaises au XV siècle, 106-110.

11. Les Cent nouvelles nouvelles, éd. Franklin P. Sweetser (Genève : Droz, 1996). 
autour de 1460 à la cour de Bourgogne à l'imitation des Cent nouvelles de Boccace $^{12}$ et qui ont connu dès les années 1480 une fortune imprimée considérable, laquelle a perduré tout au long des années $1530^{13}$. En 1549, ces Cent nouvelles nouvelles remaniées - dont le titre promet au lecteur une sorte de compendium à usage conversationnel (" facétieux devis ») — avaient manifestement pour vocation de donner une actualité et un lustre nouveaux à une collection quelque peu vieillie, au moment même où les Cent nouvelles de Boccace, autre best-seller des années 1530, venaient de faire l'objet d'une traduction nouvelle ${ }^{14}$. Le caractère commercial de la collection de La Motte Roullant ne fait donc guère de doute, et semble entièrement confirmé par les rééditions nombreuses - souvent plagiaires - dont elle fera l'objet ${ }^{15}$ : Plaisantz et facetieux devitz (Rouen : Robert et Jean Dugort, 1554), Joyeuses Adventures et Plaisant [sic] facetieux Deviz (Lyon : Benoît Rigaud et Jean Saugrain, 1555), Recueil des plaisantes et facétieuses nouvelles (Lyon : Eustache Barricat, 1555 ; Anvers : Gérard Spelman, 1555) ${ }^{16}$.

Derrière l'auteur lui-même, ce «Seigneur de La Motte Roullant Lyonnais », pourrait se cacher nul autre que Jean Réal lui-même. C'est du moins l'hypothèse de Roger Dubuis, qui voyait dans le nom même de La Motte Roullant une anagramme en forme d'aveu ${ }^{17}$ : «Le mot toullant Réal », Réal « toullant » le mot, c'est-à-dire "dérobant " les devis, pillant le recueil initial. Notons encore que la mention de l'origine lyonnaise de ce « docte auteur » est, dans l'hypothèse

12. Selon le titre couramment attribué au Décaméron au Moyen Âge et à la Renaissance, en français (Cent nouvelles) comme en italien (Cento novelle).

13. Sur la fortune des Cent nouvelles nouvelles, voir, outre les travaux de Georges Dubuis - Les Cent nouvelles nouvelles et la tradition de la nouvelle en France au moyen âge (Grenoble : Presses universitaires de Grenoble, 1973), le volume collectif dirigé par Jean Devaux et Alexandra Velissariou, Autour des Cent nouvelles nouvelles, Sources et rayonnements, Contextes et interprétations (Paris : Honoré Champion, 2016).

14. Traduction d'Antoine Le Maçon : Giovanni Boccaccio, Le Decameron de M. Iean Bocace Florentin, nouvellement traduict d'Italien en Françoys par Maistre Anthoine Le Maçon conseiller du Roy et tresorier de l'extraordinaire de ses guerres (Paris : Étienne Roffet, 1545).

15. À commencer par l'édition qui paraît dès 1550 chez Jean Longis et Jean Réal : Les Facetieux deviz des cent et six Nouvelles, Nouvelles, tres recreatives et fort exemplaires pour reveiller les bons et joyeux esprits Francoys, veues et remises en leur nature.

16. Voir Pérouse, Nouvelles françaises au XVe siècle, 106-110.

17. Roger Dubuis, « Traduttore, traditore : l'affaire La Motte Roullant », in Conteurs et romanciers de la Renaissance. Mélanges offerts à Gabriel-André Pérouse, éd. James Dauphiné et Béatrice Périgot (Paris : Honoré Champion, 1997), 173-187. 
d'une « création d'atelier $»^{18}$, particulièrement intéressante, puisque toutes les premières éditions sont imprimées à Paris. Lyon et sa production éditoriale pouvaient-elles promettre un intérêt particulier à l'acheteur, ou encore suggérer une orientation stylistique précise?

Mais c'est ici la suite du péritexte qui retiendra particulièrement notre attention, en raison, notamment, de la place de choix qu'elle réserve au lecteur. L'édition ${ }^{19}$ s'ouvre, en effet, sur quatre pièces liminaires :

1. Le privilège royal, d’abord, octroyé par Henri II pour cinq ans, à « Jehan Real, imprimeur de nostre ville de Paris " pour une édition explicitement présentée comme une réédition corrigée et augmentée : "Jehan Real [...] lequel nous à faict dire \& remonstrer, que puis peu de temps enca, il à faict revoir, corriger \& adjouster les fascetieux devitz des cent nouvelles, nouvelles, livre plaisant \& recreatif, \& auquel plusieurs pourraient prendre recreation » (f. a i $v^{\circ}$ ). L'hypothèse d'une compilation d'éditeur semble ici explicitement confirmée.

2. Une épitre au lecteur de trois pages (f. a ii $v^{\circ}$-f. a iii $r^{\circ}$ ), intitulée "EPISTRE EXORTATIVE / Aux lecteurs de bonne volunté » directement adressée au lecteur et qui retiendra par la suite toute notre attention.

3. Un huitain de décasyllabes (f. a iiii $\mathrm{r}^{\circ}$ ) surmonté du titre «AV LECTEVR, RIME / à plaisir », qui souligne, comme le privilège, la finalité récréative du recueil : "Qui vouldra en brief des nouvelles scavoir, / Convient me lire, c'est cela qu'il fault veoir : Car si voulez passer le temps \& rire, Sans blasonner, ou mocquer, ne mesdire, Nouvelles sont, qui ne sont laides à veoir [...]. »

4. Une table (f. a iiii $v^{\circ}$ ), enfin, qui offre au lecteur un aperçu synoptique des nouvelles, selon un usage bien établi dans les recueils de nouvelles français : «CY COMMENCE LA TABLE / des cent Nouvelles, Nouvelles, en laquelle est de-/clairé amplement ce qui est traicté au con-/tenu du present opuscule, \& le tout / selon l'intitulation des / chapitres. »

L'orientation commerciale de l'édition et sa destination à un public large se manifestent donc dans toutes les pièces du péritexte. Tout un arsenal rhétorique est déployé pour appâter le client du libraire, promouvoir les qualités

18. Anne Réach-Ngô, éd., Créations d'atelier. L'éditeur et la fabrique de l'œuvre à la Renaissance (Paris : Classiques Garnier, 2014).

19. Nous fondons notre analyse sur l'édition imprimée à Paris, pour Jean Réal en 1549, conservée à la Bibliothèque Nationale de France sous la cote RES P-Y2-3144 (Universal Short Title Catalogue [USTC] $\mathrm{n}^{\circ}$ 52757). 
des textes qui lui sont donnés à lire, et mettre en avant les différents usages du livre, lecture récréative ou réemploi des anecdotes pour deviser en société.

\section{"Aux lecteurs de bonne volunté » : une épître sous le signe de l'intertextualité}

Parmi les différentes pièces liminaires, l'épître exhortative adressée " aux lecteurs de bonne volunté ${ }^{20}$ présente les éléments les plus intéressants pour l'analyse et doit être relevée du simple fait de son ampleur. Si les recueils de nouvelles des années 1530 commençaient timidement à intégrer des prologues, ceux-ci étaient souvent plus brefs ${ }^{21}$, et réservaient une moindre place au lecteur ${ }^{22}$. Des contre-exemples remarquables sont à signaler, tels que l'épître au lecteur de la traduction du Décaméron par Le Maçon, en 1545, ou encore, dans le domaine de la narration longue, les prologues des romans rabelaisiens ${ }^{23}$. La traduction du Décaméron par Antoine Le Maçon est en effet pourvue d'un péritexte particulièrement riche, composé d'une longue épître dédicatoire du traducteur à Marguerite de Navarre, d'une seconde épître dédicatoire en italien d'Emilio Ferretti, homme de cour italien, d'un dizain " Au lecteur », puis, enfin, d'une épître au lecteur, signée cette fois du libraire, Étienne Roffet, définissant les enjeux littéraires, linguistiques et éditoriaux de la traduction publiée. Dès 1532, la première édition du Pantagruel ${ }^{24}$ inaugurait elle-même

\section{L'épître est reproduite intégralement en annexe.}

21. Voir par exemple le bref prologue du Parangon de nouvelles honnestes et delectables a tous ceulx qui desirent veoir et ouyr choses nouvelles [...] (Paris : s.n., 1531) ; (Lyon : Denis de Harsy pour Romain Morin, 1531). Édition critique par Gabriel-André Pérouse (Genève : Droz, 1979). Le prologue, sans titre, souligne l'agrément de l'ouvrage et propose une brève réflexion sur la légitimité et la nécessité de la récréation.

22. Alors que le prologue du Parangon est tout entier consacré à souligner l'intérêt de l'ouvrage pour le lecteur, et mentionne même la forme portative du recueil « pour plus facillement en tous lieux en avoir la fruytion totale », le lecteur lui-même n’est jamais explicitement mentionné.

23. Des prologues d'envergure peuvent être signalés auparavant : on peut songer aux Illustrations de Gaule et singularités de Troie de Jean Lemaire de Belges (Paris : G. de Marneff, 1512), rééditées en 1549 à Lyon par Jean de Tournes, à une date contemporaine des Fascetieux devitz de La Motte Roulant ; on peut également mentionner le «prologue de l'acteur » de l'édition précédente du Decameron: Livre de Cameron (Paris : A. Vérard, 1485), rééditée à plusieurs reprises jusqu’en 1545. Dans le prologue de cette édition, réécriture substantielle du prologue de la traduction manuscrite de Laurent de Premierfait qui la précède, la place réservée au lecteur est toutefois plus que discrète.

24. François Rabelais, Pantagruel (Lyon : Claude Nourry, [ca. 1532]). 
une nouvelle tradition péritextuelle, en faisant entrer en scène un lecteur herméneute autorisé et complice, double pantagruéliste de la figure auctoriale elle-même ${ }^{25}$. Or, Décaméron et geste rabelaisienne fonctionneront justement comme des intertextes de premier plan dans l'épître de La Motte Roullant, et c'est d'abord pour ses accents rabelaisiens que l'édition a été mentionnée par la critique ${ }^{26}$. Le ton rabelaisien de l'épître de La Motte Roullant frappe, en effet, dès les premières lignes, et la mise en scène des publics et des lectorats convoque immédiatement l'univers des préfaces rabelaisiennes :

Tresillustres, magnificques et treschevaleureux capitaines, gentilz hommes, Courtisans, et vous autres bons souldars, qui voluntiers vous adonnez à toutes gentillesses, et souvent employez vostre jeunesse à deviser joyeusement avecques les dames et Damoyselles, pour lesquelles le plus souvent vous contrefaictes les transiz. A vous premierement j'ay dedié ces belles nouvelles, pour vous exciter à rire et gaudir gallentement, et par similitudes et faictz modernes escriptz en ce petit traicté, pour tousjours augmenter voz creditz et gaillardisses, et pour alonger les beaulx propos qu'avez accoustumé tenir avecques voz mignonnes, et gorgiases valentines. Enquoy le plus souvent vous passez les nuictz, fuyant melancolies, en beuvant d'autant pour mieulx respirer au proffit du corps, et laisse de votre memoire. A vous aussi tresvenerables beuveurs, tondeurs de nappes, crocheteurs de flascons, et autres qui voluntiers vous adonnez à scavoir nouvelles pour en faire vostre proffit es lieux celebres des vinipotatiques tavernes, et beaulx cabaretz attendant le vin fraiz, et grosses endouilles sallées, et aussi pour passer le temps, et la pluye, et de peur de dormir. ${ }^{27}$

Impossible de ne pas entendre ici les échos aux «Tres illustres et Treschevaleureux champions $»^{28} \mathrm{du}$ Pantagruel ou aux " buveurs tresillustres et vérolez

25. Voir, entre autres, Michel Jeanneret, Le Défi des signes, Rabelais et la crise de l'interprétation à la Renaissance (Orléans : Paradigme, 1994), tout particulièrement 75 et suivantes.

26. Notamment par Pérouse, Nouvelles françaises au $X V^{e}$ siècle, 106-110, qui propose une lecture très stimulante de l'épître de La Motte Roullant.

27. Seigneur de la Motte Roullant, Les Fascetieux devitz des cent nouvelles, nouvelles, tres recreatives et fort exemplaires pour resveiller les bons espritz Françoys (Paris : Jean Réal, 1549), f. a ii vº.

28. François Rabelais, Pantagruel, "Prologue de l'auteur », in Euvres complètes, éd. Mireille Huchon (Paris : Gallimard, 1994), 213. Dorénavant désigné à l'aide du sigle OC, suivi du numéro de la page. 
tresprécieux ${ }^{29} \mathrm{du}$ Gargantua, et de ne pas percevoir, plus généralement, le ton du bateleur propre à ces romans à l'encan analysés par Ariane Bayle ${ }^{30}$. Le jeu avec l'intertexte rabelaisien contribue ainsi à l'élection d'un lectorat défini d'abord par sa capacité, précisément, à déchiffrer les références intertextuelles, et à se reconnaître dans la communauté des lecteurs, amateurs de bons passetemps et bons pantagruélistes, fondée par les romans rabelaisiens. Mais dans la perspective qui est la nôtre, on sera attentif surtout à une désignation du lectorat qui joue sur l'identification du lecteur à un type social, en quoi elle se démarque, précisément, des prologues rabelaisiens. Les « gentilshommes » et « courtisans » des premières lignes renvoient, en effet, au milieu de la cour, tout comme ces « dames et damoyselles » qui apparaissent comme les destinataires secondes de ces nouvelles, via leur transmission orale " $^{31}$ ( pour alonger les beaulx propos qu'avez accoustumé tenir avecques voz mignonnes, et gorgiases valentines ${ }^{32}$ ). Il faudrait commenter chaque mot de ces premières lignes et de l'épître entière, qui s'amuse à servir au lecteur tout un condensé de topoï et de thèmes caractéristiques de la rhétorique péritextuelle des recueils narratifs, tout en multipliant les références intertextuelles. Soulignons pour l'instant l'insistance et la variété de ces apostrophes aux lecteurs, qui impriment au texte son rythme oratoire et, par un effet de liste, esquissent toute une galerie de portraits de lecteurs, auxquels le lecteur " réel » était invité à s'identifier. Poursuivons-en brièvement l'inventaire.

Après les "gentilzhommes et courtisans " à qui les nouvelles sont explicitement dédiées ( $\mathrm{A}$ vous premierement j’ay dedié ces belles nouvelles»), l'auteur interpelle les «tresvenerables beuveurs, tondeurs de nappes, crocheteurs de flascons ", fréquentant " tavernes et cabarets ", qui évoquent là encore bien sûr Rabelais, mais désignent aussi, à travers la référence intertextuelle, un tout

29. François Rabelais, Gargantua, "Prologue de l'auteur », in OC, 5. Des deux prologues, c'est le premier, le prologue du Pantagruel, qui présente les similitudes les plus évidentes avec l'épître de La Motte Roullant.

30. Ariane Bayle, Romans à l'encan. De l'art du boniment dans la littérature du XVIe siècle (Genève : Droz, 2009).

31. Même si elle est empruntée à Rabelais (voir note suivante), la distinction entre les lecteurs - masculins — et les auditrices — féminines — est remarquable. Faut-il y voir un retournement misogyne, facétieux ou non, du topos de la destination féminine des recueils de nouvelles boccaciens? 32. Voir Rabelais, Pantagruel, «Prologue de l'auteur », OC, 213 : "y avez maintesfoys passé vostre temps avecques les honorables Dames et Damoyselles, leur en faisans beaulx et longs narrez [...] dont estez bien dignes de grande louange, et memoire sempiternelle ». 
autre lectorat, plus viril, moins courtisan et, osons le mot, plus populaire. Ce sont ensuite les « beaulx mignons parasites, escornifleurs et autres fleureteurs de grosses cuisines qui tant honnestement faictes vostre proffit des nouvelles de court » qui sont désignés, donc à nouveau des courtisans, mis en scène cette fois dans leur rapport aux puissants, et en particulier aux clercs, allusion peut-être à ces amuseurs professionnels et écrivains de cour qui "vivaient de leur plume » et de la protection des grands :

A vous beaulx mignons parasites, escornifleurs et autres fleureteurs de grosses cuisines qui tant honnestement faictes vostre proffit des nouvelles de court pour resjouyr ses gros evesques, abbez, chanoynes et autres personnages farciz de benefices qui vous recoivent en leurs tables. ${ }^{33}$

Enfin, l'épître fait référence aux « gouteux et verollez qui jadis [ont] tant virilement $[\ldots]$ combatu ${ }^{34}$, c'est-à-dire aux soldats et officiers revenus de guerre, qui devaient représenter une réalité sociale bien présente en ces années où les guerres d'Italie battaient encore leur plein : "A vous aussi gouteux et verollez qui jadis tant virilement avez combatu, que maintenant en portez les enseignes et guidons de peur que ne soyez oysifz $[\ldots] »^{35}$. Tous ces lecteurs se trouvent réunis dans une longue énumération finale qui étoffe et diversifie encore le public interpellé à chaque apostrophe, et mêle les qualifications sociales à des considérations physiques et médicales, pour souligner de manière ludique l'universalité du livre et de ses usages possibles :

Et generalement à toutes personnes de quelque qualité et condition qu'ilz soient sains et malades, bossus, boyteux, et contrefaictz, à tous barbiers, marchans, hostes, hostesses et lingeres, quecunque prebstres, moynes jeunes et vieulx, et autres enragez de rien faire. ${ }^{36}$

Certes, il ne s'agit pas de prendre à la lettre cette énumération ludique qui égrène, autant que des catégories sociales de lecteurs, des caractères et des types

33. Seigneur de la Motte Roullant, Les Fascetieux devitz des Cent nouvelles nouvelles, f. a ii vo-f. a iii $\mathrm{r}^{\circ}$. 34. Voir là encore Rabelais, Pantagruel, «Prologue de l'auteur », OC, 214 : " Mais que diray je des pauvres verolez et goutteux?».

35. Seigneur de la Motte Roullant, Les Fascetieux devitz des Cent nouvelles nouvelles, f. a ii vo-f. a iii $\mathrm{r}^{\circ}$. 36. Seigneur de la Motte Roullant, Les Fascetieux devitz des Cent nouvelles nouvelles, f. a iii ro. 
littéraires. En effet, le lecteur de nouvelles ne manquera pas de reconnaître dans ces " treschevaleureux capitaines ", " dames et damoyselles ", ou "prêtres " et " marchands », le personnel même des récits qui lui sont donnés à lire, conformément à l'une des lois génériques de la nouvelle, qui veut que les personnages soient caractérisés d'abord par leur appartenance à un type social : « bourgeoise », " gentilhomme », « curé ». Il n'y a qu'à considérer la table (f. a iiii $v^{\circ}$ ) même des Fascetieux devits pour s'en convaincre et pour retrouver, dans la liste des titres de " chapitres ", des types sociaux très proches de ceux qui sont énumérés dans l'épître : "un financier amoureux de la femme de son voysin " (nouvelle 1), un « frere cordelier borgne » (nouvelle 2), « un gentilhomme qui fist accroire à sa musnière, que son penil luy tombait » (nouvelle 3), ou encore, dans le désordre, un " mercier », un « bourgeois ", un "noble chevalier », un " yvrogne ». Avant de chercher des référents extra-fictionnels à la joyeuse troupe de lecteurs esquissée dans l'épître, il s'agit donc de faire toute sa part aux jeux de déplacements internes qui se manifestent entre l'espace intradiégétique des nouvelles et l'espace extra-diégétique de l'épître, voire, comme le suggère la présence de ces dénominations dans la table des nouvelles, entre les deux pièces péritextuelles que constituent la table et l'épître. N'oublions pas que nous avons probablement affaire à une «création d'atelier $»^{37}$, orchestrée par le libraire Jean Réal lui-même, et qu'il était très fréquent que les imprimeurs et libraires déplacent, dans ce type d'entreprise éditoriale, le matériau textuel de leur source d'un endroit de l'œuvre à un autre ${ }^{38}$.

Il n'en reste pas moins que la référence extra-fictionnelle de cette galerie de lecteurs mérite réflexion, ne serait-ce que parce que l'auteur joue savamment

\section{Réach-Ngô, Créations d'atelier.}

38. Sans doute faut-il aussi rappeler que la porosité entre espace intra- et extra-diégétique constitue une autre loi générique de la nouvelle, et que celle-ci se manifeste, du Décaméron aux Cent nouvelles nouvelles bourguignonnes, par la présence d'un récit-cadre (ou de son esquisse, dans le second cas, à travers la mention des conteurs de nouvelles). Là encore, on peut se demander si les Fascetieux devitz ne procèdent pas à un transfert d'éléments intra-diégétiques - la mise en scène de la réception des nouvelles dans le récit-cadre - vers l'espace extra-diégétique de l'épître. Dans le recueil de nouvelles de tradition boccacienne, la fiction-cadre offre déjà une représentation du public, à travers l'évocation de la ronde des conteurs qui récitent et écoutent les nouvelles. Or, dans la « joyeuse compagnie " des conteurs du Décaméron, "gentilshommes " et "nobles dames » figurent en bonne place. Ainsi, les " gentilshommes » et " courtisans » de notre épître, contant de « beaux propos " aux « dames » et " gorgiases valentines ", pouvaient évoquer assez précisément l'auditoire féminin des recueils de tradition boccacienne pour le lecteur des Fascetieux devitz. 
de la confusion entre fiction et référence historico-sociale, et que l'identification de cette dernière est constitutive de la force comique même de l'épître.

\section{Publics de cour et publics de taverne}

Pour délicate qu'elle soit, la lecture socio-historique de cette galerie de lecteurs conduit alors à distinguer, derrière l'évocation, deux types de publics : d'un côté, le public des milieux de cour, à travers la figure du " courtisan " et de son pendant féminin, la " gorgiase valentine $»^{39}$, qui ouvrent le texte et le traversent en réalité de part en part (le texte se clôt sur une invitation à devenir le «parangon des dames ») ; de l'autre, un lectorat plus populaire et plus viril, celui du public des tavernes, auquel on pourrait ajouter le clergé - qui, effectivement, constitue l'une des cibles privilégiées de la littérature narrative (on songera simplement aux moines de l'Heptaméron qui se cachent derrière une haie pour écouter les nouvelles que se racontent les devisants).

À chacun de ces publics, ou presque, est associée une désignation de l'œuvre qui leur est destinée : " belles nouvelles " pour les courtisans (" gentilz hommes, Courtisans [...], à vous premierement j’ay dedié ces belles nouvelles ») ou encore «nouvelles de court » («à vous beaulx mignons parasites, escornifleurs et autres fleureteurs de grosses cuisines ») ; "fascetieux devitz » pour les vénérables buveurs et les goutteux et vérolés qui ont jadis virilement combattu ("A vous aussi gouteux et verollez qui jadis tant virilement avez combatu [...], j’ay dédiée [sic] ces fascetieux devitz »).

La distinction des publics se double donc d'une distinction générique et semble délimiter deux lectorats que divisent leurs goûts, leurs lectures, mais aussi

39. Alors que le terme de «valentin » désigne manifestement l'amant dans l'épître, avec une connotation de frivolité, « valentine » renvoie à son équivalent féminin, l’amante, la demoiselle courtisée. Ces sens ne sont pas répertoriés dans les dictionnaires : Le Dictionnaire de l'ancienne langue française de Godefroy donne pour «valentin » : «marchand de bijoux et de petites nippes que les galants donnaient à leurs maîtresses " (Dictionnaire de l'ancienne langue française, éd. numérique Classiques Garnier, https:// www.classiques-garnier.com/numerique-bases/godefroy). Edmond Huguet donne pour l'adjectif « valentin »: " relatif aux valentins, sorte de fêtes galantes. » (Edmond Huguet, Dictionnaire de la langue française du XVI siècle, éd. numérique Classiques Garnier, https://www.classiques-garnier.com/ numerique-bases/huguet). Le terme connote donc à la fois l'artifice, la mise en scène galante, mais aussi le commerce, ce qui confirme pleinement la dimension satirique de l'évocation. Il en va de même pour l'adjectif « gorgias, gorgiase », plus courant, et auquel Huguet donne le sens de « joli, gracieux, élégant, bien vêtu». 
l'usage qu'ils en font : les «belles nouvelles » sont destinées aux conversations galantes et à la séduction des dames ("pour alonger les beaulx propos qu'avez accoustumé tenir avecques voz mignonnes $»^{40}$ ), alors que les « facétieux devis » sont faits pour alimenter les propos de table et séjours en taverne des « tondeurs de nappes » et " crocheteurs de flacons » (" pour en faire vostre proffit es lieux celebres des vinipotatiques tavernes, et beaulx cabaretz attendant le vin fraiz »). Remarquons que la distinction n'est pas seulement sociale mais aussi genrée, ce qui ne surprendra guère en ces années où la Querelle des Amyes occupe le devant de la scène littéraire, et divise dans d'autres recueils célèbres - on pense bien sûr à l'Heptaméron - lecteurs et lectrices selon leurs goûts et leurs aspirations.

D’un point de vue stylistique, ces deux tendances génériques, nouvelle et facétie (ou "facétieux devis »), correspondent bien, toutefois, à deux orientations majeures de la narration brève de l'époque, et renvoient sans doute aussi à des modes de circulation orale distincts dans des contextes de sociabilité divers. On observera une fois de plus ici l'habileté de l'éditeur qui réunit ces deux orientations stylistiques dans le titre (Les Fascetieux devitz des cent nouvelles, nouvelles : nous soulignons) pour les dissocier ensuite plaisamment dans l'épître («nouvelles de court » vs. « fascetieux devitz»), par une mise en concurrence de deux lectorats et de deux publics dont l'objectif premier est de séduire la clientèle la plus large.

De ces deux publics-cibles, c'est assurément le premier, le lectorat « de cour ", qui est le plus aisé à saisir. En effet, la « nouvelle de cour » semble désigner un phénomène littéraire et social qui se définit à la fois par un milieu de production et de réception, un ensemble de traits génériques et stylistiques, et une sociabilité courtoise à laquelle elle semble indissociablement liée. Le succès de la nouvelle dans les milieux aristocratiques est bien attesté par l'historiographie littéraire, et les documents conservés (catalogues de bibliothèques, récits historiques, dédicaces) témoignent notamment de l'importance du Décaméron de Boccace dans ce qui semble devenir une véritable mode littéraire, couronnée en 1545 par l'édition de la traduction d'Antoine Le Maçon, trésorier du roi, commanditée par la sœur de François Ier Marguerite de Navarre ${ }^{41}$. L’ancrage

40. On trouve encore, plus bas dans l'épître : "à fin qu'en apres le tout leu vous soyez digne d'estre appellé le parangon des dames, beau deviseur, et le plaisant Valentin ».

41. Voir, entre autres, Jean Balsamo, «Le Décaméron à la cour de François Ir », Op. cit. 7 (1996) : 231-239; Mireille Huchon, "Caméron et Décaméron: de l'influence du Boccace travesti à la française ", 
aulique du genre a notamment été étudié par Jean Balsamo, qui montre que la réception française du Décaméron est, dans les années 1540, un phénomène lié à la cour de François $\mathrm{I}^{\text {er }}$, dans lequel le roi et sa sœur ont joué un rôle de premier $\operatorname{plan}^{42}$. Les imprimeurs-libraires qui ont édité le fleuron le plus célèbre de cette tradition, la traduction du Décaméron par Antoine Le Maçon, se sont d'ailleurs empressés d'afficher cet ancrage aulique en multipliant les signes péritextuels qui exhibent cette destination prestigieuse : privilège royal, dédicaces à Marguerite de Navarre, épître de l'éditeur soulignent conjointement le prestige et la destination aristocratique de l'entreprise éditoriale. Quelques années plus tard, le prologue de l'Heptaméron témoignera à son tour de la place occupée par la nouvelle boccacienne dans les milieux de cour, modèle à imiter, parangon littéraire, et pratique sociale : en des lignes souvent citées ${ }^{43}$, Parlamente, l'une des conteuses de l'Heptaméron, fait explicitement référence au Décaméron de Boccace, loué par François I ${ }^{\text {er }}$ et son entourage, en invitant ses compagnons à « en faire autant que Boccace » et à conter à leur tour cent nouvelles, définissant ainsi explicitement l'Heptaméron comme un nouveau Décaméron.

Si la «nouvelle de cour » renvoie donc d'abord à un milieu de production et de réception, elle se définit également par son inscription dans une forme de sociabilité mise en scène dans les recueils eux-mêmes. Le recueil de Boccace déjà inscrivait la récitation de nouvelles dans un ensemble de passe-temps aristocratiques évoqués dans le récit-cadre et placés sous le signe des Muses (danses, chants, promenades et conversations), passe-temps dont la nouvelle apparaissait comme une modalité parmi d'autres. Quelques années avant la publication des Fascetieux devitz, la traduction du Courtisan de Baldassar

in Boccaccio e le letterature romanze tra Medioevo e Rinascimento, atti del Convegno internazionale Boccaccio e la Francia, 19-20 maggio 2003, Firenze-Certaldo, éd. Simonetta Mazzoni Peruzzi (Florence : Alinea, 2006), 57-82.

42. Balsamo, «Le Décaméron à la cour de François $\mathrm{I}^{\mathrm{er}}$ », 231-239.

43. Marguerite de Navarre, L’Heptaméron des nouvelles, éd. Nicole Cazauran (Paris : Gallimard, 2000), 65-66 : «Entre autres je croy qu'il n'y a nulle de vous qui n'ait leu les cent nouvelles de Jean Bocace, nouvellement traduictes d'Italien en François : desquelles le Roy treschrestien François premier de ce nom, monseigneur le Daulphin, ma dame la Daulphine, ma dame Marguerite ont faict tant de cas, que si Bocace du lieu où il estoit les eust peu ouïr, il eust deu rescusciter à la louenge de telles personnes. A l'heure j'ouy les deux dames dessus nommées avec plusieurs autres de la court qui se deliberoient d'en faire autant, sinon en une chose différente de Boccace, c'est de n'escrire nouvelle, qui ne fust veritable histoire». 
Castiglione $(1537)^{44}$ vient donner une assise théorique à cet usage de la nouvelle, puisque le Courtisan emprunte à Boccace une grande part des exemples qui viennent alimenter les développements théoriques sur l'art de la conversation de l'homme de cour. La nouvelle devient, à la suite du traité de Castiglione, un agrément de la vie de cour, et les références appuyées de l'épître de La Motte Roulant aux « nouvelles de cour » et aux « devis » des « courtisans » flattant les puissants et séduisant les "mignonnes valentines " pourraient bien être une référence directe à cette pratique sociale et littéraire. La désignation générique du recueil des Fascetieux devitz (qualifié d'« opuscule » et de " petit traité ») prend alors tout son sens : en promettant à ses lecteurs des anecdotes prêtes à l'emploi, destinées à alimenter les conversations galantes des gentilshommes et de leurs valentines, le compendium de La Motte Roullant s'inscrit dans la continuité directe de la réception réservée aux nouvelles de Boccace dans le Courtisan ${ }^{45}$.

À cette nouvelle de cour, dont les contours se dessinent avec une certaine précision, et qui associe, dans son champ notionnel, une tradition générique et un lectorat déterminé, notre épître oppose une autre tradition, celle des « facétieux devis ", qui renvoie à son tour à une tradition orale, celle des « colloques et des banquets où s'échangent mets et mots ${ }^{46}$, et pour laquelle la Renaissance est là encore réputée représenter un âge d'or ${ }^{47}$. Le pendant livresque de cette tradition, le recueil de facéties, connaît en ces années un succès croissant ${ }^{48}$, que

44. La première traduction, attribuée à Jacques Colin, paraît en 1537 à Lyon, chez Denis de Harsy sous le titre Les quatre livres du Courtisan du Conte Baltazar de Castillon. Reduyct de langue Italicque en Françoys. François Juste imprimera en 1538 une version corrigée de ce texte comportant une épître de Dolet à Mellin de Saint-Gelais.

45. Tout en la détournant dans une direction facétieuse teintée de misogynie : il n'y a pas de symétrie entre hommes et femmes dans la transmission des nouvelles suggérée par l'épître, contrairement à ce que l'on observe dans le Décaméron. Voir note 31.

46. Huchon, «Introduction », in Rabelais, OC, XI.

47. Voir, entre autres, Michel Jeanneret, Des Mets et des mots (Paris : José Corti, 1987), et plus récemment, Nicolas Kiès, Rencontrer en devisant. La conversation facétieuse dans les recueils bigarrés des années 1580 (Du Fail, Cholières, Bouchet) (thèse de doctorat, Université Paris 4, 2015).

48. Sur le développement de la facétie en France, on signalera, outre les travaux fondateurs de Lionello Sozzi (en particulier : «Les Facezie di Poggio nel Quattrocento francese », in Miscellanea di studi e ricerche sul Quattrocento francese [Torino : Giappichelli, 1967], 409-507) et de Henri Weber (en particulier : "La facétie et le bon mot de Pogge à Des Périers", in Humanism in France at the End of the Middle Ages and in the Early Renaissance, éd. A.H.T. Levi [Manchester-New York : 1970], 82-10), les travaux plus récents du groupe FACEF coordonné par Dominique Bertrand («Fortunes et Avatars de l’Esprit Facétieux entre France et Italie de la fin du Moyen-Âge à l'Âge Classique », MSH Clermont-Ferrand, 
confirmera bientôt l'une de ses réalisations les plus accomplies, les Nouvelles récréations et joyeux devis de Bonaventure des Périers (Lyon : Granjon, 1558). Au début des années 1540, elle est essentiellement représentée par les Facéties du Pogge ${ }^{49}$ et par les Cent nouvelles nouvelles bourguignonnes, qui connaissent, l'un et l'autre, une fortune éditoriale durable. Depuis leur traduction française par Guillaume Tardif dans les années $1490^{50}$, les Facéties de Pogge sont régulièrement rééditées, à Lyon et à Paris, sous des titres qui soulignent déjà la destination orale et conversationnelle du genre, et qui exerceront une influence durable sur l'évolution du récit bref français. Citons seulement les éditions lyonnaises des Facéties, imprimées dans les années 1530 à Lyon par Olivier Arnoullet: Les facecies de Poge florentin translatees de latin en francoys qui traictent de plusieurs nouuelles choses moralles / Pour racompter en toutes bonnes compaignies ${ }^{51}$.

Plus nombreuses encore sont les éditions des Cent nouvelles nouvelles bourguignonnes, qui, en dépit de leur lien intertextuel explicite avec les Cent nouvelles de Boccace $^{52}$, semblent étroitement associées, au XVI ${ }^{e}$ siècle, à la tradition facétieuse fondée par Le Pogge. L'essentiel des éditions des Cent nouvelles nouvelles est imprimé cette fois à Paris, depuis l'édition princeps

2013-2014), ceux de l'équipe « Facéties » du Labex OBVIL coordonnée par Louise Amazan et MarieClaire Thomine (Université Paris-Sorbonne), ainsi que les thèses récemment soutenues ou en cours : Nicolas Kiès, Rencontrer en devisant. La conversation facétieuse dans les recueils bigarrés des années 1580 (Du Fail, Cholières, Bouchet) (thèse de doctorat, Université Paris-Sorbonne, 2015) ; Tiphaine Rolland, Le "vieux magasin " de Jean de La Fontaine. Les Fables, les Contes et la tradition européenne du récit plaisant (XVe-XVII s.) (thèse de doctorat, Université Paris-Sorbonne, 2017) ; Louise Amazan, La mise en livre des recueils facétieux entre la fin $d u X V^{e}$ et le milieu $d u X V I^{e}$ siècle (thèse de doctorat, Université Paris-Sorbonne, en cours).

49. Poggio Bracciolini, Liber Confabulatiorum, Florence, Biblioteca Medicea Laurenziana, ms. 14381453 : Le Pogge, Facéties. Confabulationes, éd. et trad. Stefano Pittaluga et Etienne Wolff (Paris : Les Belles Lettres, 2005).

50. Guillaume Tardif, Les Facecies de Pogge, Traduction du Liber facetiarum de Poggio Bracciolini, éd. Frédéric Duval et Sandrine Hériché-Pradeau (Genève : Droz, 2003).

51. Les facecies de Poge florentin translatees de latin en francoys qui traictent de plusieurs nouuelles choses moralles (Lyon : Olivier Arnoullet, s.d. [ca. 1530 et 1532]). La première de ces éditions est conservée à la BnF sous la cote Res Y2 1085 ; la seconde à la Bibliothèque de l'Arsenal sous la cote $8^{\circ}$ B30471 Rés. Seule la seconde présente le sous-titre « Pour racompter en toutes bonnes compaignies ».

52. Au-delà du titre, qui fait explicitement référence aux Cent nouvelles de Boccace, le prologue de l'auteur définit le recueil comme une imitation du Décaméron. Voir Les Cent nouvelles nouvelles, éd. Sweetser, 22. 
pour Antoine Vérard en 1486, jusqu'aux éditions des années $1530^{53}$, qui ont servi de source à la compilation de La Motte Roullant. On remarque toutefois aussi quelques éditions lyonnaises, là encore pour Olivier Arnoullet, où les Cent nouvelles nouvelles paraissent sous un titre qui souligne la parenté avec les Facéties du Pogge et suggère l'inscription dans un programme éditorial commun : Les cent nouuelles [sic]. S'ensuyvent les cent nouvelles contenant cent hystoires / ou nouveaulx comptes plaisans / a deviser en toutes bonnes compaignies par maniere de ioyeusetés4.

La formule « à deviser en toutes bonnes compagnies » reprend quasiment à l'identique le titre des Facéties ( «à raconter en toutes bonnes compaignies ») : la destination conversationnelle du recueil narratif est déjà explicite. Du soustitre des Cent nouvelles nouvelles au titre de Fascetieux devitz des Cent nouvelles nouvelles il n'y avait alors qu'un pas, que le compilateur Jean Réal franchit allégrement en radicalisant encore l'inscription du recueil dans une circulation orale : les « comptes plaisans à deviser » deviennent « devis ».

Ainsi, les «facétieux devis » mentionnés à plusieurs reprises dans l'épître constituent bien, sinon un contrepoint générique, au moins une variante distincte des «belles nouvelles » de tradition boccacienne, même si la frontière entre « nouvelle » et "facétie », tradition boccacienne et facétieuse, est loin d'être étanche et que l'épître les associe notamment dans une même vocation orale et conversationnelle ${ }^{55}$. Peut-on aller au-delà de cette distinction générique

53. Par exemple : S'ensuyvent les Cent nouvelles (sic), contenant cent hystoires ou nouveaux comptes plaisans, a deviser en toutes bonnes compaignies par manière de ioyeuseté (Paris : Veuve Jean Trepperel, s.d. [ca. 1530]).

54. Deux éditions au moins : Les Cent nouvelles (sic). S'ensuyvent les cent nouvelles contenant cent hystoires ou nouveaulx comptes plaisans a deviser en toutes bonnes compaignies par maniere de ioyeuseté (Lyon : Olivier Arnoullet, s.d. [ca. 1530] et Les Cent nouvelles. S'ensuyvent les cent nouvelles contenant cent hystoires nouveaulx qui sont moult plaisans a racompter en toutes bonnes compaignies par maniere de ioyeuseté (Lyon : Olivier Arnoullet, 1532).

55. Rappelons que le Courtisan lui-même emprunte au Décaméron bon nombre de nouvelles facétieuses : le développement de la nouvelle, en France et en Italie, est inséparable de celui de la facétie, et le recueil de Boccace a joué un rôle fondateur dans l'histoire du genre facétieux. Il faut néanmoins constater que se développe en France, dans le sillage du Décaméron, une tradition narrative d'une veine distincte de la facétie, illustrée par exemple par les nouvelles sentimentales de Jeanne Flore (Comptes amoureux [Lyon : Denis de Harsy, ca. 1531]), ou encore, plus tardivement dans le siècle, par les Comptes du monde adventureux de l'anonyme «ADSN» (Paris : Jean Longis, Vincent Sertenas, Etienne Groulleau, 1555). Le prologue de ce recueil met en scène une compagnie de jeunes nobles en voyage, écourtant le chemin par des récits « enrichis d'amoureuses recontres ", qui rappellent de près la situation de sociabilité conteuse 
et associer à la tradition facétieuse un lectorat précis, un public déterminé ? Si le public de «cour» semble bien renvoyer à une réalité socio-historique identifiable, force est de constater que le lectorat des "facétieux devis » constitue un groupe plus hétérogène et plus insaisissable, caractérisé avant tout par un lieu de fréquentation commun, les «tavernes » et les « cabarets », et par des traits de comportement, l'oisiveté et le goût de la bonne chère. Sans doute l'intertextualité littéraire pèse-t-elle plus lourd encore dans l'évocation de ce second lectorat, qui se résume peut-être entièrement à la référence rabelaisienne, de sorte que c'est surtout de manière indirecte et implicite que l'on peut dégager certains traits de ce lectorat facétieux : un lectorat hétérogène, viril, comprenant soldats et abbés, essentiellement défini par opposition au lectorat de cour, c'està-dire excluant de sa ronde la compagnie honnête des dames.

Malgrél'« effet de réel » suscité par la joyeuse troupe de lecteurs qui déferle dans notre épître, la référence de l'évocation se dérobe partiellement à l'analyse autant qu'elle impose sa présence bariolée et pittoresque au seuil de l'ouvrage. Brouillant les frontières entre fiction et réalité socio-historique, accumulant les distinctions et les dichotomies pour les rendre aussitôt caduques, l'épître oriente et désoriente. Au demeurant, elle jouait sans doute parfaitement son rôle auprès du lecteur, lequel devait reconnaître, derrière ces " mignons courtisans » et " crocheteurs de flacons », sinon des figures de lecteurs, du moins des traditions génériques et des pratiques sociales qu'il devait s'amuser de voir si plaisamment évoquées. Il reste alors à déterminer quelle place lui était désignée dans ce dispositif, et si, entre lecteur de cour et convive de taverne, il était amené à choisir son camp. La différenciation des figures de lecteur dans l'épître contribue-t-elle à l'élection d'un public aux dépens d'un autre? Permet-elle l'émergence d'une communauté de lecteurs qui transcenderait les oppositions esquissées?

\section{Le lecteur idéal de l'épître exhortative : élection et construction d'un public de nouvelles « français»}

Fidèle à la rhétorique publicitaire du bonimenteur, cette épître au lecteur - c'est un premier point à noter - ne divise pas explicitement ces différents publics,

évoquée dans notre épître (Comptes du monde adventureux [Paris : E. Groulleau, 1555], « Epître », f. 4 $\mathrm{v}^{\circ}$ ). Bien que liées dans leur histoire et souvent associées dans les recueils, nouvelle facétieuse et nouvelle sentimentale correspondent bien à des tendances génériques et stylistiques distinctes. 
mais prétend au contraire les rassembler, en promettant un livre convenant à tous, propre à tout dire et à tout faire, rejoignant là encore l'une des mythologies de la geste rabelaisienne : "rajeusnir les vieulx emerites, [faire] rire les piteux, allume[r] le feu de joye, [faire] croistre l'encre au cornet, [servir] d'emplastres au besoing, et [faire] oublier toutes rencunes, maledictions et injure », telle est la longue liste des effets bénéfiques du livre ${ }^{56}$. On a également souligné que la destination orale unifie la réception du recueil, et permet de concilier les usages et les contextes différents dans lequel il sera utilisé : conversation virile des tavernes, propos alanguis des Valentins aux Valentines.

Si l'une des finalités du prologue est donc incontestablement de réunir les catégories de lecteurs et les pratiques de lecture les plus irréconciliables pour promouvoir le livre, il se dégage néanmoins l'impression que l'ironie railleuse de notre docte auteur lyonnais ne traite peut-être pas avec équité ses différents lecteurs, et que sa verve se dirige plus contre les mignons parasites et valentins des dames, qui sont en réalité moqués tout au long du texte, que contre les buveurs vérolés amateurs de contes à rire, auxquels l'auteur nous invite finalement à nous joindre, à la toute fin du texte, nous « suppliant de boire ».

Une nouvelle ligne de partage se dessine ainsi, non plus entre des publics socialement distincts, mais entre bons lecteurs, maîtrisant le jeu de l'intertextualité et sachant rire de tout, et lecteurs naïs, attendant " louanges immortelles » et "dragées et confitures ${ }^{57}$ de leurs valentines ${ }^{58}$, en échange de leurs «belles nouvelles $»^{59}$.

Or, le titre du recueil de notre auteur prétendument lyonnais (ville de Rabelais jusqu'en 1535, et lieu d'édition de ses premiers ouvrages ${ }^{60}$ ) annonce

56. Là encore, les échos avec le prologue du Pantagruel, qui énumère lui aussi les nombreuses vertus thérapeutiques du livre, sont nombreux.

57. Voir la fin de l'épître : « En quoy faisant, pourrez acquerir louanges immortelles, avecques dragées et potz de confitures que vous leur demanderez si estes si hardy, vous suppliant de boire, in sempiterna secula. Ainsi soit. "Voir le prologue du Pantagruel, OC, 213, passage cité note 32 : " dont estez bien dignes de grande louange, et memoire sempiternelle».

58. Lecteurs naïfs ou, au contraire, lascifs... Les dons attendus des "valentines ", " dragées » et autres " pots de confiture », peuvent sans aucun doute se lire aussi dans un sens sexuel.

59. Plus haut dans l'épître : «J'ay presenté ces belles nouvelles ».

60. Les premiers éditeurs du Pantagruel et du Gargantua sont lyonnais : Claude Nourry pour le Pantagruel, s.d. [1532], François Juste pour le Gargantua, s.d. [1535]. Plusieurs rééditions paraissent à Lyon au début des années 1540, juste avant la publication des Fascetieux devitz. Voir Huchon, «Pantagruel, Note sur le texte », in Rabelais, OC, 1228 et « Gargantua, Note sur le texte », in Rabelais, OC, 1056. 
un livre destiné à " réveiller les bons espritz françoys ", à quoi l'épître fait écho en développant tout un argumentaire sur le goût et le naturel des lecteurs français. En évoquant les raisons qui l'ont poussé à rééditer les Cent nouvelles nouvelles bourguignonnes, et à en livrer une version élaguée, l'auteur de l'épître explique en effet :

Et pour ceste occasion, ce livre avoit esté delaissé comme chose ennuyeuse et mal limée au gré et plaisir des Françoys, lesquelz sur toutes nations appettent brieveté en leurs langaiges, au moyen de leur tant subtil entendement, comprenant en brief la substance du narré tant sont industrieux et bien nez. (f. a iii $\mathrm{r}^{\circ}$ )

À qui d'autre opposer ces «bons esprits français » et « lecteurs de bonne volonté » qu'à ces amateurs de nouvelles à l'italienne, qui vantent à la même époque Boccace et son style courtisan et copieux ? Rabelais n'est jamais nommé dans cette épître exhortative, pas plus que Boccace. Il semble cependant qu'une mise en concurrence discrète de ces deux maîtres structure le texte de La Motte Roullant, et qu'à travers eux, deux traditions, l'une italianiste et boccacciophile, l'autre française, facétieuse et rabelaisante, sont mises en concurrence ${ }^{61}$. Les développements sur le naturel français et le goût pour la brieveté peuvent en effet faire penser aux arguments de vente déployés dans l'édition du Décaméron de 1545 , dans un contexte, précisément, d'émulation nationale et d'affirmation du génie de la langue française. L'éditeur de la traduction du Décaméron, Étienne Roffet, expliquait lui aussi les raisons qui l'avaient amené à traduire à nouveau un recueil qui avait auparavant été «mis en pièce et en lopins ${ }^{62}$ par des traducteurs « qui avaient tant mal besogné qu'il n'est possible de plus $»^{63}$. Or, la nouvelle traduction du Décaméron promet à ses lecteurs des

61. Les échos intertextuels aux romans de Rabelais, d'une part, et à la nouvelle boccacienne, d'autre part, sont difficiles à nier. Soulignons toutefois que ces oppositions ne sauraient s'ériger en système : la copia rabelaisienne, comme le goût de Boccace pour la facétie " de taverne ", compliquent considérablement la lecture du phénomène très particulier d'anti-italianisme que nous croyons pouvoir déceler dans ce prologue.

62. Boccaccio, Le Decameron de M. Iean Bocace Florentin, « Estienne Roffet dit le Faulcheur, aux lecteurs ", fol. a $\mathrm{v} \mathrm{r}^{\circ}$.

63. Boccaccio, Le Decameron de M. Iean Bocace Florentin, " A treshaute et tresillustre princesse Marguerite de Navarre », fol. a ii $\mathrm{r}^{\circ}$. 
qualités stylistiques qui sont aux antipodes du modèle esquissé par l'épittre des Fascetieux devitz, et mettent en avant le "français si bon, si courtisan » de la traduction, et l'« abondance » d'une langue aussi « copieuse » que celle de Boccace. Si l'on garde à l'esprit que les Fascetieux devitz viennent offrir une nouvelle vie à une collection, les Cent nouvelles nouvelles, qui faisait longtemps concurrence aux Cent nouvelles de Boccace dans le paysage éditorial français, ne peut-on pas penser que l'édition de La Motte Roullant «Lyonnais » était délibérément chargée de damer le pion à la nouvelle traduction française de Boccace, imprimée à Paris, à une époque où la décaméromania faisait rage à la cour de France ? L'ironie railleuse qui guide la plume de notre docte Lyonnais semble pour le moins à rapprocher du courant anti-italianiste qui s'exprimera bientôt sous la plume d'une nouvelle génération de poètes, eux aussi soucieux d'illustrer - et de définir - une Muse française.

En publiant en 1549 une version modernisée des Cent nouvelles nouvelles, l'éditeur faisait alors coup double : il profitait de la vogue des nouvelles à l'italienne évoquées dans le prologue, et proposait à son lecteur un produit éditorial convenant mieux à son « naturel » français. Il prolongeait et ravivait ainsi une tradition narrative présentée comme ancestrale (" antique ", selon le mot de l'épître) celle des Cent nouvelles nouvelles, tout en lui attribuant une nouvelle figure titulaire et une portée nouvelle : celle d'une herméneutique et d'une éthique de la lecture, comme le voulait le modèle rabelaisien. La rhétorique de l'épître réactivait ainsi une rivalité entre nouvelle italienne et française qui s'inscrit aux origines même du genre en France ${ }^{64}$, et dont jouait déjà le prologue du recueil bourguignon, source de l'édition de Jean Réal. Le lecteur quant à lui ne pouvait que s'amuser de cette rivalité facétieuse, et choisir peut-être son camp : il n'y a guère de raison de douter que les différentes orientations éditoriales et génériques qui lui étaient présentées dans cette épître ne correspondent à des goûts distincts, perceptibles dans les cercles de lettrés comme dans les boutiques de libraires. Il reste que notre éditeur-bonimenteur - habileté commerciale ou souci réel de la fortune de son livre - ménage généreusement un accès au texte à tout lecteur potentiel sachant rire, plaisantin valentin ou vérolé très précieux. En ceci, l'épître joue pleinement son rôle de captatio benevolentioe, nouvelle au Moyen Âge (Paris : Honoré Champion, 2006), 21-28. 
tout en opérant le seul tri qui vaille au seuil d'un recueil facétieux : lecteurs « de bonne volonté », bienvenus. Fâcheux, s’abstenir.

\section{Annexe \\ Epistre exortative Aux lecteurs de bonne volunté ${ }^{65}$}

Tresillustres, magnificques et treschevaleureux capitaines, gentilz hommes, Courtisans, et vous autres bons souldars, qui voluntiers vous adonnez à toutes gentillesses, et souvent employez vostre jeunesse à deviser joyeusement avecques les dames et Damoyselles, pour lesquelles le plus souvent vous contrefaictes les transiz. A vous premierement j'ay dedié ces belles nouvelles, pour vous exciter à rire et gaudir gallentement, et par similitudes et faictz modernes escriptz en ce petit traicté, pour tousjours augmenter voz creditz et gaillardisses, et pour alonger les beaulx propos qu'avez accoustumé tenir avecques voz mignonnes, et gorgiases valentines. Enquoy le plus souvent vous passez les nuictz, fuyant melancolies, en beuvant d'autant pour mieulx respirer au proffit du corps, et laisse de votre memoire. A vous aussi tresvenerables beuveurs, tondeurs de nappes, crocheteurs de flascons, et autres qui voluntiers vous adonnez à scavoir nouvelles pour en faire vostre proffit es lieux celebres des vinipotatiques tavernes, et beaulx cabaretz attendant le vin fraiz, et grosses endouilles sallées, et aussi pour passer le temps, et la pluye, et de peur de dormir. A vous beaulx mignons parasites, escornifleurs et autres fleureteurs de grosses cuisinescuisines qui tant honnestement faictes vostre proffit des nouvelles de court pour resjouyr ses gros evesques, abbez, chanoynes et autres personnages farciz de benefices qui vous recoivent en leurs tables. A vous aussi gouteux et verollez qui jadis tant virilement avez combatu, que maintenant en portez les enseignes et guidons de peur que ne soyez oysifz, j'ay dédiée ces fascetieux devitz, lesquelz font de leur

65. Seigneur de la Motte Roullant, Les Fascetieux devitz des cent nouvelles, nouvelles, tres recreatives et fort exemplaires pour resveiller les bons espritz Françoys (Paris : Jean Réal, 1549), f. a i vo-f. a iii rº. Nous reproduisons ici l'épître de notre édition de référence. La transcription respecte l'orthographe et la ponctuation d'origine. Seules les modernisations d'usage ont été pratiquées : dissimilation $\mathrm{i} / \mathrm{j}$ et $\mathrm{u} / \mathrm{v}$, résolution du tilde, remplacement de l'esperluette par « et ». 
naturel, rajeusnir les vieulx emerites, font rire les piteux, allument le feu de joye, font croistre l'encre au cornet, servent d'emplastres au besoing, et font oublier toutes rencunes, maledictions et injures, car elles ont esté tainctes au fleuve de l'esté. Et generalement à toutes personnes de quelque qualité et condition qu'ilz soient sains et malades, bossus, boyteux, et contrefaictz, à tous barbiers, marchans, hostes, hostesses et ligeres, quecunque prebstres, moynes jeunes et vieulx, et autres enragez de rien faire. J'ay presenté ces belles nouvelles, desquelles furent jadis escriptes en tresrude et mal consonant langaige, avecques raisons prolixes, et le plus souvent sans propos, enquoy les auditeurs estoient treschargez pour la grande multitude et variété de parolles. Et pour ceste occasion, ce livre avoit esté delaissé comme chose ennuyeuse et mal limée au gré et plaisir des Françoys, lesquelz sur toutes nations appettent brieveté en leurs langaiges, au moyen de leur tant subtil entendement, comprenant en brief la substance du narré tant sont industrieux et bien nez. Et pour ceste cause quand ma tresaymée Valentine nous auroit produict en sa faveur, nous l'avons diligemment veu et leu et totallement immué le langaige anticque et remis les nouvelles en leur naturel, brief et succinct, comme vous pourrez veoir par la lecture d'icelles, à fin que tant vos oreilles qu'espritz ne recoivent choses inutiles et mal consonantes aux fascetieux devitz. Et davantage, pour ce que es anticques j'ay trouvé plusieurs comptes indignes de voz oreilles, qui estoient aussi sans saulces ne raisons. Nous les avons ostées, au lieu desquelles avons mis plusieurs comptes tresjoyeulx et dignes d'estre refferez es assemblées et en toutes bonnes compaignies, à propos pour eviter melancolie et faire trouver le vin meilleur, desquelles plusieurs comme j'espere en lecheront leurs doidz, et n'en auront à demy, mais pour ceste heure ilz se pourront contenter en demourant sur leur appetit, esperant qu'alors que je les voiray fameliques, \& appetans autres semblables, je les resasiray encores de meilleures que je leur garde en ma manche bien serrée de peur qu'ils ne s'en vollent, car le danger y est grand. Doncques, tresdebonnaires, je vous supplie que ces fascetieux devitz vous lisiez et iceulx retenez de bonne affection, au travail de vostre corps en vostre noble memoire, et vous mesmes montrez envers les dames et autres bonnes compagnies qu'en avez entendu quelque chose, à fin qu'en apres le tout leu vous soyez digne d'estre appellé le parangon des dames, beau deviseur, et le plaisant valentin. Enquoy faisant, pourrez acquerir louanges immortelles, avecques dragées et potz de confitures que vous leur demanderez si estes si hardy, vous suppliant de boire, in sempiterna secula. Ainsi soit. 